\title{
Amyloidosis presenting as a colonic mass. A case report
}

\author{
Lara Dakhoul • Hesam Keshmiri • Asem Werdi • \\ Brian J. Blumenstein
}

Accepted: 5 August 2014 / Published online: 21 August 2014

(C) Springer-Verlag Berlin Heidelberg 2014

\section{Purpose}

Amyloidosis refers to a group of disorders characterized by accumulation of amyloid, a fibrillary protein, in various organs. However, amyloidosis presenting as a colonic mass or "amyloidoma" without evidence of systemic disease is uncommon, and its clinical presentation can mimic those of a colonic neoplasm. We present a rare case of an old lady with rectal bleeding that turned to be caused by an amyloidoma.

\section{Case}

A 90-year-old female with multiple medical comorbidities presented from a nursing home for dizziness, presyncopal episode, and bright red blood per rectum. Laboratory data revealed hemoglobin of 6.7 and international normalized ratio (INR) of 2.5. Initial resuscitation was performed and the patient was stabilized with hydration, blood transfusions, and fresh frozen plasma (FFP). A colonoscopy was performed which revealed a malignant-appearing, ulcerated, submucosal $4 \times 5 \mathrm{~cm}$ mass in mid transverse colon with a blood clot on the surface. A Biopsy was taken showing benign colonic tissue, ulcerated colonic mucosa, and acute inflammatory exudates. Based on its malignant appearance and the high risk of ongoing GI bleed, laparoscopic resection of the transverse colon was performed. The pathology reported an ulcerated mass with heavy deposits of amyloid on Congo red stain involving lamina propria, submucosa, and muscle wall with no evidence of malignancy. Special stains were positive for light chains,

L. Dakhoul $(\bowtie) \cdot$ H. Keshmiri • A. Werdi • B. J. Blumenstein Department of Internal Medicine, University of Illinois/Advocate Christ Medical Center, 4440 W 95th St, Oak Lawn, IL 60453, USA e-mail: laragirl_10@hotmail.com indicating ulceronodular localized amyloidosis (amyloidoma) [1]. Thirteen pericolonic lymph nodes were negative for malignancy. Postoperatively, the patient had complicated hospitalization course. After she recovered in the intensive care unit (ICU), she was discharged on hospice.

\section{Discussion}

Amyloidosis presenting as a colonic mass is very rare. The complications can be as aggressive as a neoplasm, such as bleeding, intestinal infarction, and ischemic colitis. Reported presentations are various and include abdominal pain, rectal bleeding, weight loss, watery diarrhea, and pseudo-obstruction [2]. In this case, the mass turned out to be an isolated amyloidoma despite the initial endoscopic biopsy being negative. It is important in this situation to determine whether amyloidosis is local versus systemic as the management and the prognosis are quite different.

In conclusion, any solitary lesion of the colon may not always be what it appears on colonoscopy [3]. Microscopic examination results can be surprising [4]. Therefore, one should always consider the possibility of amyloidoma.

\section{References}

1. Parks RW, O' Rourke D et al (2002) Perforation of the sigmoid secondary to localized amyloidosis. Ulster Med J 71(2):144-146

2. Ellen CE, Nagar M (2008) Gastrointestinal manifestations of amyloidosis. Am J Gastroenterol 103:776-787

3. Chen J-H, Lai S-J et al (2002) Localized amyloidosis mimicking carcinoma of the colon. Am J Roentgenol 179:536-537

4. Watanabe T, Kato K et al (1999) A case of solitary amyloidosis localized within the transverse colon presenting as a submucosal tumor. Gastrointest Endosc 49(5) 